\title{
Hot-Electron Electroluminescence Under RF Operation in GaN-HEMTs: A Comparison Among Operational Classes
}

\author{
Tommaso Brazzini, Michael A. Casbon, Michael J. Uren, Member, IEEE, Paul J. Tasker, Fellow, IEEE, \\ Helmut Jung, Hervé Blanck, and Martin Kuball, Senior Member, IEEE
}

\begin{abstract}
Electroluminescence microscopy and spectroscopy are used to compare the average hot-electron concentration and temperature under radio frequency (RF) operation class A, class B, and class $F$ modes. From the results obtained, class $A$ results, on average, in the highest hot-electron concentration, while class $F$ is the mode with the lowest concentration due to its "L"'shaped load line. The electron temperature extracted from the electroluminescence spectra is reduced with increasing RF power, reflecting the dominance of electroluminescence from the portion of the load line in the semi-on region. The electroluminescence method is not able to give substantial information on the portion of the load line with high field and low current density which will be responsible for the potentially damaging hottest electrons present in the channel.
\end{abstract}

Index Terms-AIGaN/GaN, class A, class B, class F, electroluminescence (EL), electron temperature, hotelectrons, microwave field-effect transistor (FET), radio frequency (RF) degradation.

\section{INTRODUCTION}

A $1 \mathrm{GaN} / \mathrm{GaN}$ devices have proven themselves for commercial applications in the field of radio frequency (RF), power amplifiers, and recently power switches. Despite GaN offering excellent properties for the successful realization of high-performance devices, a full understanding of RF reliability is still lacking. Various aspects of RF reliability testing of GaN-based devices have been addressed by many groups, such as gate metal instabilities [1], inverse piezoelectric effects [2], passivation breakdown [3], and generation of trap states [4]; comparisons between dc and RF reliability testing were also performed [5]-[8]. Hot-electron degradation depends on both the concentration of hot electrons involved in the transport,

Manuscript received February 8, 2017; accepted March 15, 2017. Date of publication April 4, 2017; date of current version April 19, 2017. This work was supported by the U.K. Engineering and Physical Sciences Research Council under Grant EP/K026232 and Grant EP/K02633X. The review of this paper was arranged by Editor K. J. Chen.

T. Brazzini, M. J. Uren, and M. Kuball are with the Centre for Device Thermography and Reliability, H. H. Wills Physics Laboratory, University of Bristol, Bristol BS8 1TL, U.K. (e-mail: tommaso.brazzini@ bristol.ac.uk; Martin.Kuball@ bristol.ac.uk).

M. A. Casbon and P. J. Tasker are with the Centre for High Frequency Engineering, Cardiff University, Cardiff, CF24 3QR, U.K.

$\mathrm{H}$. Jung and $\mathrm{H}$. Blanck are with United Monolithic Semiconductors $\mathrm{GmbH}, 89081 \mathrm{Ulm}$, Germany.

Color versions of one or more of the figures in this paper are available online at http://ieeexplore.ieee.org.

Digital Object Identifier 10.1109/TED.2017.2686782 but also on the energy the electrons obtain as a result of the high accelerating electric field. The stressing of the device under high hot-electron concentrations can in fact result in the generation of defects [9]-[11].

Electroluminescence (EL), which originates from hotelectron scattering with defects and consequently Bremsstrahlung [12], is widely used for the assessment of the hot-electron-related degradation rate in GaN-based devices [13]-[16]. Recently, EL has been successfully used to understand how hot-electrons act on the device under class B and class J RF operations [8], and compared to dc operation on the same load line [9]. This was achieved under passive load-pull operation, which restricts the power levels and RF modes that can be investigated. In this paper, we gain a more complete insight covering a wider range of operational modes using accurate measurement of $\mathrm{RF}$ $I-V$ waveforms in a fully active harmonic load-pull environment using a system that allows pulsed or continuouswave $(\mathrm{CW})$ operation while simultaneously performing EL microscopy and spectroscopy [17]. The focus of this paper is to compare the device hot-electron behavior under different classes of RF operation (classes A, B, and F) with EL. Classes A, B, and F are widely used RF modes and the reliability of devices when operated under these classes in a real system is important to understand. The outcome of this work shows that class $\mathrm{A}$ is the mode generating the highest concentration of hot electrons during operation, with class $\mathrm{F}$ having the lowest at high RF powers. It is found that measured electron temperature drops with increasing RF power in all cases, despite the fact that examination of the load lines tells that peak electron temperature must be increasing. This arises because EL measures a weighted average of emission from all parts of the load line with emission from the hottest electrons swamped by emission from the much higher density of hot electrons generated in the semi-ON region. EL under $\mathrm{RF}$ conditions can be mainly used as a tool to examine degradation modes which are linked to hot-electron flux and not for any that are linked to electron temperature.

\section{EXPERIMENTAL DETAILS}

The $8 \times 125 \mu \mathrm{m}-\mathrm{AlGaN} / \mathrm{GaN}$ HEMTs studied here comprise an $\mathrm{AlGaN} / \mathrm{GaN}$ heterostructure grown on an Fe-doped 

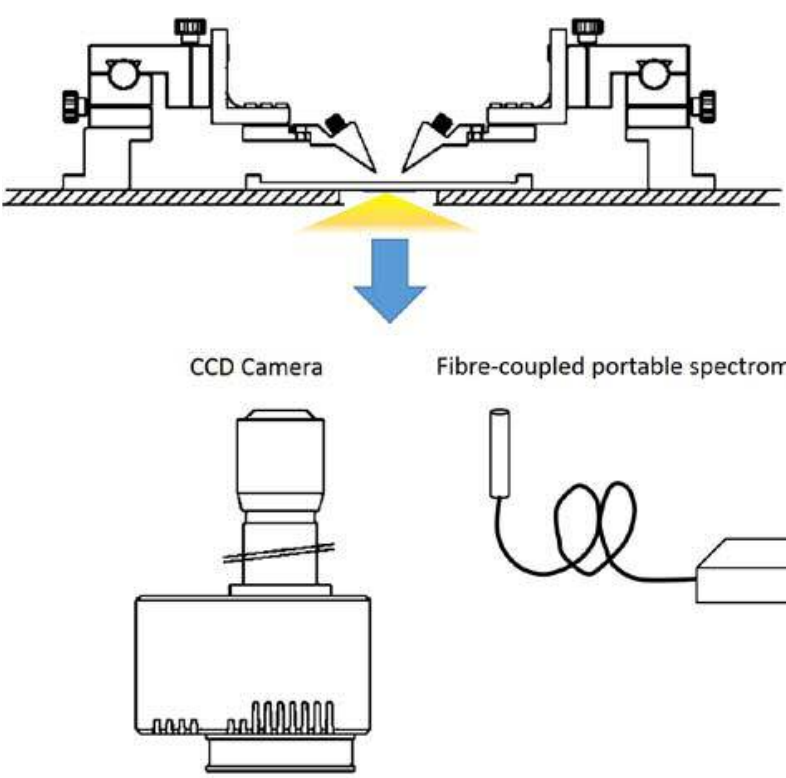

Fibre-coupled portable spectrometer

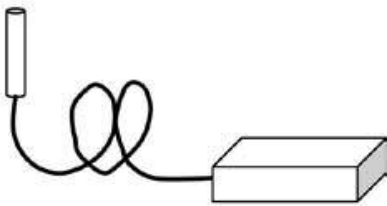

Fig. 1. Schematic of setup used for the experiments. The sample stage allows measurement with a standard load-pull system and light access to the device through the substrate, i.e., from the device backside. The light emitted from the device was collected from the bottom and sent either to the CCD camera (for EL microscopy) or to the spectrometer (for EL spectroscopy).

GaN buffer layer on a semi-insulating SiC substrate, with 0.25 $\mu \mathrm{m}$ gate length. The experimental measurement setup is shown in Fig. 1. A large signal network analyser system architecture based on a VTD SWAP-X402 receiver was used to operate the devices. The RF pulsing unit was provided through an external modulator with high-speed RF switches $(1 \mathrm{GHz})$, while modulating the drain under dc with a high-side FET switch, if required. The RF and dc drain bias can be independently switched between pulse and dc, without making any changes to the sampling regime; therefore, any measured changes can safely be ascribed to the device under test. This setup allows the full range of common classes, such as $\mathrm{A}, \mathrm{B}, \mathrm{AB}, \mathrm{F}, \mathrm{J}$, and continuous $\mathrm{F}$, to be explored in both pulse and $\mathrm{CW}$ modes [17]. The cases analyzed in this paper are classes A, B, and F. When operating in class $A$, the quiescent bias current is set to a high level of half the expected peak current, and this current is maintained independent of the output power level. In class $\mathrm{B}$, the second-harmonic load impedance is set to a short circuit and the quiescent bias current is initially set to a low level, which rises as the RF drive is increased, resulting in improved efficiency at the expense of some gain and nonlinearity. True class $\mathrm{F}$ requires the even harmonics (second, fourth, etc.) to be short circuited, while the odd harmonics (third, fifth, etc.) are presented with an open circuit. Ideally, this results in an "L"shaped dynamic load line; however at microwave frequencies, it is usually only practical to control the second and third harmonics, causing the corner to be cut, as shown in Fig. 2.

EL measurements, during the load-pull operation, were performed from the backside of the device through the transparent $\mathrm{GaN}$ layer and $\mathrm{SiC}$ substrate, enabling access to the entire source-drain region including areas underneath

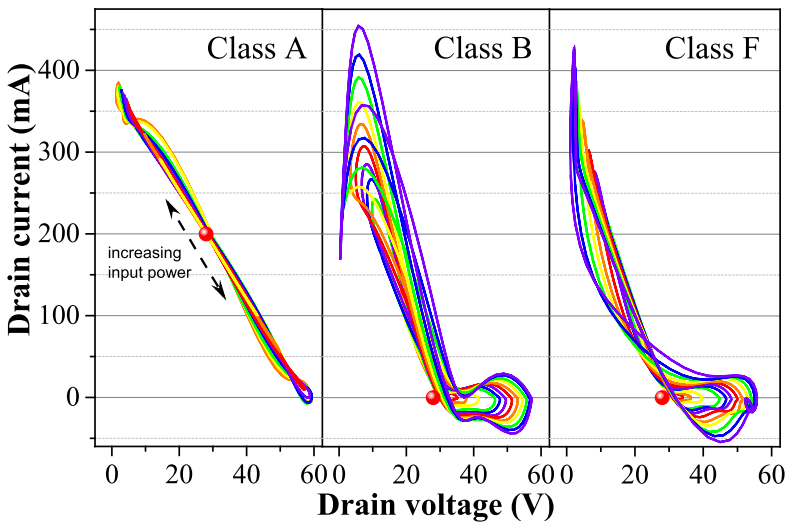

Fig. 2. Output response of the $A I G a N / G a N$ device studied under classes $\mathrm{A}, \mathrm{B}$, and $\mathrm{F}$ from 20 - to $35-\mathrm{dBm}$ output power. The quiescent dc bias points are shown in the three cases (red bullets). (a)

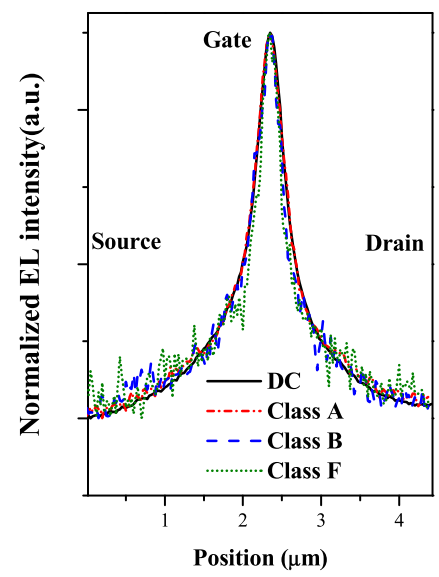

(b)

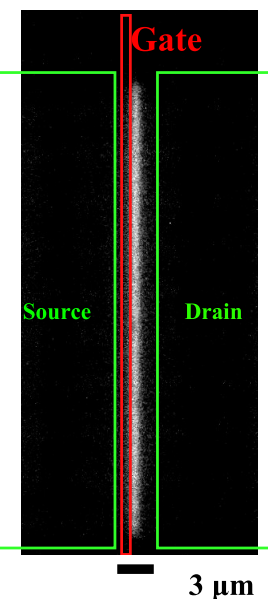

Fig. 3. (a) Normalized EL light intensity spatial distribution from an AIGaN/GaN HEMT during operation for dc $(28 \mathrm{~V}, 200 \mathrm{~mA})$ and RF classes $A, B$, and $F\left(35-d B m P_{\text {out }}\right)$. (b) EL image from a single finger of the AIGaN/GaN device under study, recorded from the backside of the wafer under dc bias. Source $(S)$, drain $(D)$, and gate $(G)$ contacts are denoted.

the metal contacts (as shown in Fig. 1). EL imaging was carried out with $50 \times$ objective and a Hamamatsu digital charge-coupled device (CCD) camera, while the spectra were recorded with a broad-spectrum fiber coupled to a compact spectrometer (Maya 2000-Ocean optics QEPro) sensitive in the range 200-1100 $\mathrm{nm}$. As the CCD camera records spectra integrated over many seconds, all EL spectra and intensities are an average over a multitude of RF cycles.

\section{REsults AND Discussion}

The output responses of the device under class A, class B, and class $\mathrm{F}$ RF operations $(1 \mathrm{GHz})$ are reported in Fig. 2 for different input powers. Fig. 3 shows the profile of the EL in the active device area under dc $(28 \mathrm{~V}, 200 \mathrm{~mA})$ and RF operation (classes $\mathrm{A}, \mathrm{B}$, and $\mathrm{F}$ ) at $35-\mathrm{dBm}$ output power, with peak emission near the gate contact, the device region with highest electric field. No substantial difference is observed for the spatial extension of the light emitted under different operating 


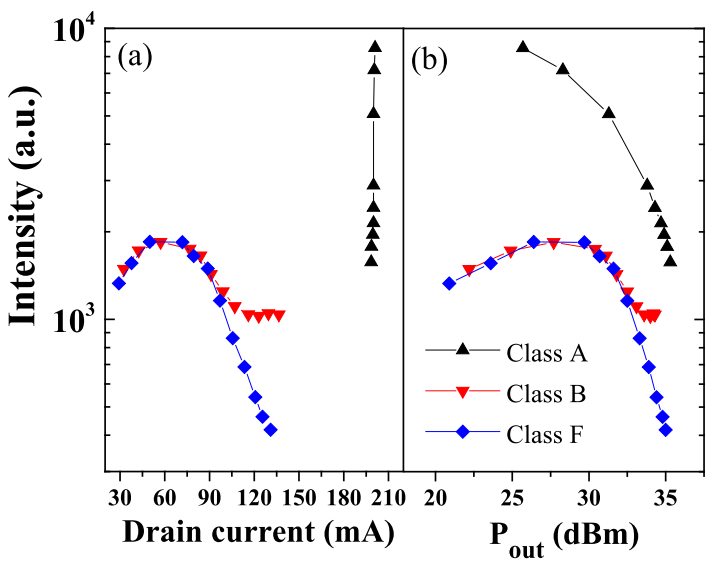

Fig. 4. EL intensity in log-scale for class A, class B, and class $F$ $\mathrm{RF}$ modes (bias point $V_{\mathrm{DS}}=28 \mathrm{~V}$ ) versus (a) average drain current and (b) output power.

conditions when the intensity is normalized. This suggests that the width of the electric field spatial distribution during RF sweeps does not change significantly compared to the dc case for drain bias of $28 \mathrm{~V}$ [18], and between the different RF modes.

As shown in Fig. 4, the EL intensity for class A mode decreases monotonically with increasing input and output power. The quiescent dc bias point when no RF power is applied $\left(V_{\mathrm{DS}}=28 \mathrm{~V}, I_{\mathrm{DS}}=200 \mathrm{~mA}\right)$ corresponds to a semiON condition and has the highest hot electron concentration, such that any increase in the input RF power will result in a decrease of the average hot-electron concentration, hence a decrease in the EL intensity. The cases of classes B and F are different: the quiescent bias point for both modes is $28-\mathrm{V}$ and 0-mA drain current (Fig. 2). They both show a bell-shaped curve with drain current and output power and a maximum at about $60 \mathrm{~mA}$ and $30 \mathrm{dBm}$. The reason for that is that at zero input RF power the device is in OFF-state, and hence, no EL is emitted because no hot-electrons are present. An increase in RF power will inject more hot electrons into the device up to a maximum point. After that the increase in the input power will push the device to work in regions of the $I-V$ planes with low density of hot electron (linear region and OFF-state region, see Fig. 2 for clarity). This is consistent with our previous results [7], [8]. On average the hot-electron concentration for the two classes ( $\mathrm{B}$ and $\mathrm{F}$ ) is always lower than class $\mathrm{A}$.

Class $\mathrm{F}$ is ideally a mode that generates no hot electrons due to the "L" shape of the load line. In this mode, the device works only in the linear regime and the nearly OFF-state, avoiding completely the semi-ON region, so no hot electrons are expected to be generated. However, as previously noted, the sweep inevitably works partially in the semi-ON region of the $I-V$ plane. That makes the EL intensity behavior similar to class $\mathrm{B}$ until the load line reaches the knee, occurring in this case at $100-\mathrm{mA}$ average drain current and $32-\mathrm{dBm}$ output power, where class $\mathrm{F}$ starts to differ substantially from class B (see Fig. 2 for a comparison). At that point, class $F$ spends less time in the semi-ON region and more time in the linear region (high current-low voltage). Consequently,

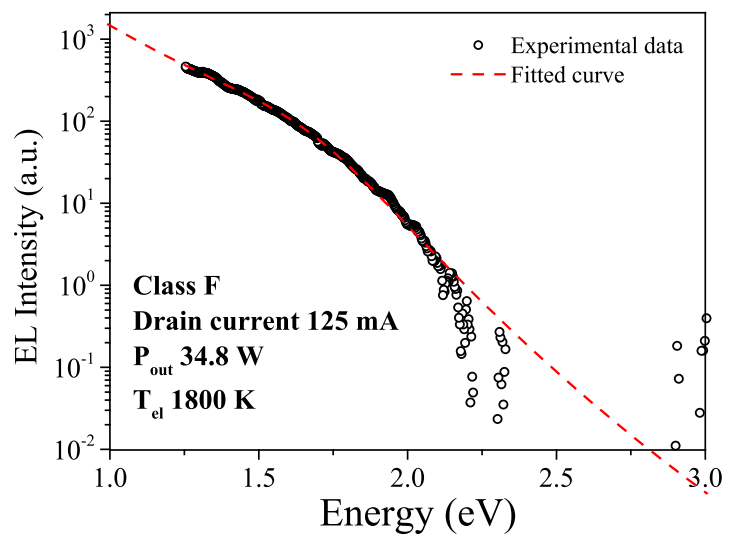

Fig. 5. Example fitting of the measured EL spectrum and extraction of the electron temperature $\left(T_{\mathrm{el}}\right)$. The fitting function is an exponential term, associated with $T_{\mathrm{el}}$, and a modulating term to take into account FabryPerot oscillations within the passivation and epitaxial layers of the device structure [12]. In the present experiment, the period of the oscillation is about $2 \mathrm{eV}$, and hence in the graph, only one maximum is visible.

the EL intensity, and hence the hot-electron concentration, decreases dramatically with respect to class B (see Fig. 4).

The extraction of electron temperature $\left(T_{\mathrm{el}}\right)$ from the measured EL spectrum can be performed by fitting the following equation [12]:

$$
I_{\mathrm{EL}} \sim M_{\mathrm{FP}} \times \exp \left(-E_{\mathrm{photon}} /\left[k_{B} T_{\mathrm{el}}\right]\right)
$$

to the experimental data where $M_{\mathrm{FP}}$ is a modulating factor taking into account the effects of interference due to the multilayer structure of the sample, $I_{\mathrm{EL}}$ is the EL intensity, $E_{\text {photon }}$ the photon energy, and $k_{B}$ the Boltzmann's constant. In this sample, the interference effect $\left(M_{\mathrm{FP}}\right)$ was associated with the passivation layer. The result of the fitting is shown in Fig. 5. It is important to note that the measured $T_{\mathrm{el}}$ actually represents a weighted average electron temperature over the full 1-ns cycle of the load line.

Fig. 6 displays the results of the $T_{\mathrm{el}}$ obtained for the three classes analyzed under different average dc current and output power. We neglected here for simplicity the lattice temperature rise which at maximum corresponds to $10 \%$ of the electron temperature. Fig. 7 shows the power-added efficiency and peak channel temperature for varying output power for each mode. Any increase in input power strongly reduces the $T_{\mathrm{el}}$ measured in all cases. The reason for that mostly resides in the way the device electrically sweeps along the load line. Since the semi-ON parts of the load lines have the highest EL intensity, as discussed previously, the measured EL spectrum under $\mathrm{RF}$ is mostly dominated by these semi-ON regions. With increasing input power the $T_{\mathrm{el}}$ behavior will therefore be dominated by lower field regions in the $I-V$ plane. Once saturated, less time is spent in the semi-ON part of the $I-V$ plane and so that peak EL emission intensity shifts to lower $V_{\mathrm{ds}}$.

For the same output power values, class $\mathrm{A}$ has lower $T_{\mathrm{el}}$ compared with the other two classes, related to the lower proportion of the complete RF cycle that the device spends in the high-field regions (high drain voltage) of the $I-V$ plane (see Fig. 8 for visual explanation). As noted earlier, 


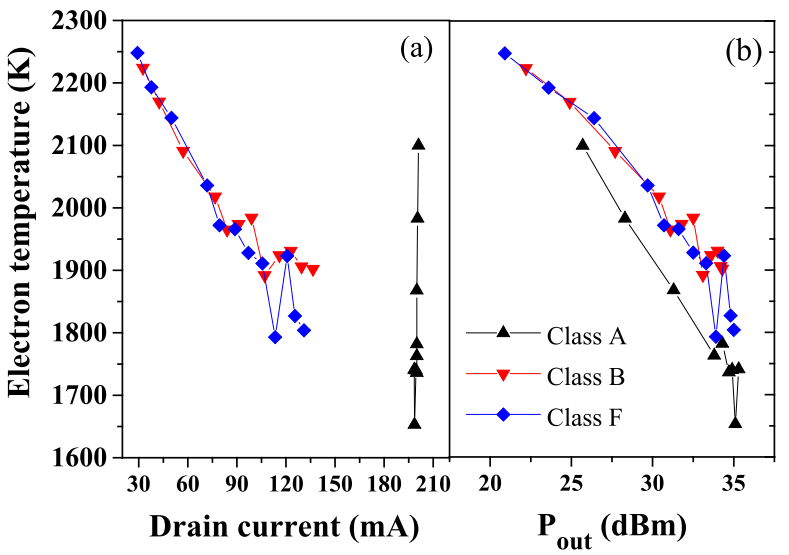

Fig. 6. Electron temperature extracted from the EL spectra for the three RF modes reported versus (a) average drain current and (b) output power.

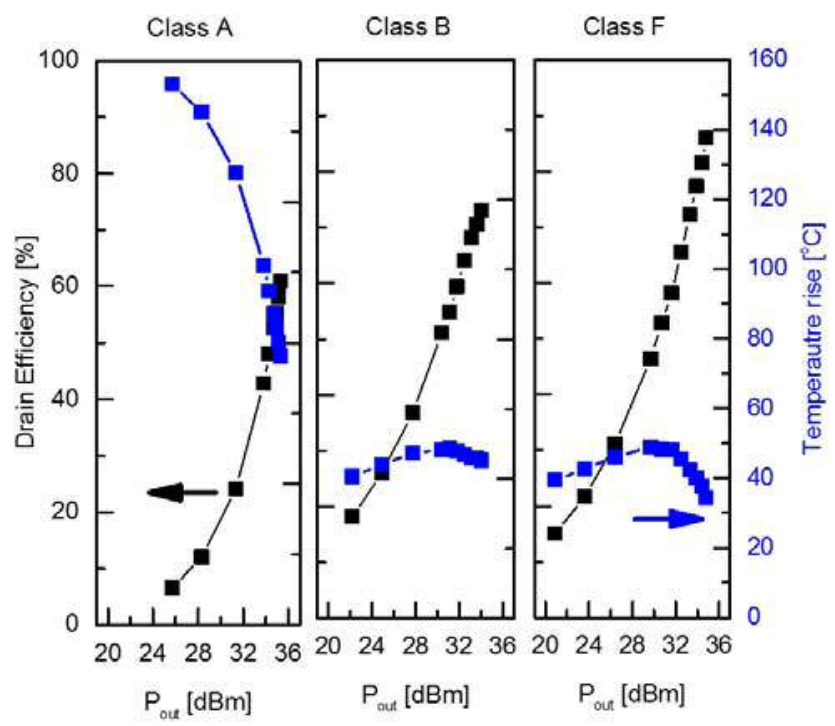

Fig. 7. Drain efficiency and peak channel temperature determined from experimentally validated thermal simulations, for classes $\mathrm{A}, \mathrm{B}$, and $\mathrm{F}$ as a function of RF output power.

this is a weighted average value temperature over a 1-ns sweeping RF cycle. The EL intensity (Fig. 4) and measured electron temperature (Fig. 6) both decrease with increasing $\mathrm{RF}$ power. However, the peak electron temperature is not measured and is likely to increase with RF power since a portion of the load line would enter the high-field, low current regime.

Similar considerations apply to the case of classes B and F. They show very similar $T_{\mathrm{el}}$ measurements until the highest powers, and both show slightly higher values than class A. Both reduce with input power (drain current or $P_{\text {out }}$ ). This can be explained by examining region 3 of the $I-V$ plane swept during class $\mathrm{A}$, class $\mathrm{B}$, and class $\mathrm{F}$ operations, as highlighted in Fig. 8. The load lines spend a large proportion of the time in the higher field region where there is almost no EL emission (zero/low current), which naturally does not contribute to the measured EL spectrum. At the highest output power, the measured class $\mathrm{B} T_{\mathrm{el}}$ is highest, which is consistent with the load line loop at peak $V_{\mathrm{DS}}$, which has larger average drain current with respect to class $F$.

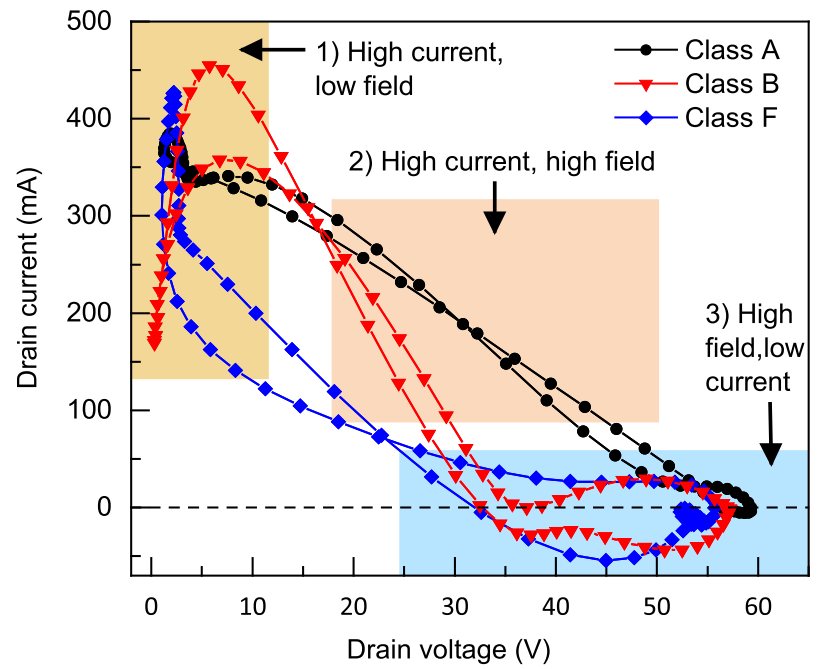

Fig. 8. Representation of the most important regions of degradation in the $I-V$ plane during RF operations overlaid with the three classes of load lines measured at the same output power of $35 \mathrm{dBm}$. The highest density of hot electrons is generated in region (2), whereas the hottest electrons are generated in region (3). High-field degradation can occur in region (3). Note boundaries between regions are for illustration purposes only and should not be considered as abrupt.

From a reliability point of view, considering the EL intensity results, class A is potentially more affected by hot electrons, and hence, hot-electron degradation is more likely to occur when compared to classes B and F. When both classes B and F are run at high driving powers, the hot-electron concentration under class F is lowest. (Class B has double the EL intensity of class F.) This is a direct consequence of the particular shape of the class $\mathrm{F}$ sweep in the $I-V$ plane after reaching the knee $\left(P_{\text {out }}>30 \mathrm{dBm}\right)$. Due to this fact, class $\mathrm{F}$ is the $\mathrm{RF}$ mode in which less hot-electron degradation is expected to occur when the devices are operated at high input powers. On the other hand, electric field peak values can also be important when considering degradation effects. Classes B and F can potentially suffer from enhanced field-induced degradation as illustrated in Fig. 8. Even if the weighted average $T_{\mathrm{el}}$ is relatively low, there are points on the load line with very high field [19] (e.g., blue region in Fig. 8) that will not be detected with EL spectroscopy because of the negligible EL intensity. Although class A operation can result in a higher probability of hot-electron-related degradation, field-induced degradation is less likely to occur until full power. At maximum power (as seen in Fig. 8 at $35 \mathrm{dBm}$ ), all three modes have similar high-field behavior and are likely to have broadly similar fieldinduced degradation.

\section{CONCLUSION}

The comparison of the hot-carrier behavior under three classes of RF operation (A, B, and F) is reported for $\mathrm{AlGaN} / \mathrm{GaN}$ devices. During class A operation, the device is working with considerably higher hot-electron concentrations compared to the other two modes. Hence, class A will be more affected by degradation modes which are linked to hotelectron flux. At the highest RF powers, class $F$ has the lowest EL emission consistent with its load line which largely 
avoids the semi-ON region of operation. Both classes $\mathrm{B}$ and $\mathrm{F}$ show a somewhat higher measured electron temperature than class A reflecting the fact that their load lines at low power are located in a higher field but lower current portion of the $I-V$ plane. In all classes, the electron temperature drops with increasing output power and this could be interpreted as showing that RF reduces the damage susceptibility. However, this temperature drop arises because of the weighted average nature of the measurement so that increasing power includes a larger proportion of relatively low-temperature electrons. Unfortunately, emission from hot electrons corresponding to the high voltage-low current region will be swamped by the semi-ON emission, even though the temperature of these hottest electrons will increase with RF power. Examining the measured load lines, we can infer that classes B and F may be more vulnerable to damage from this small density of hot electrons, but EL in itself cannot deliver any support to this supposition. EL is a valuable technique for dc measurement of reliability, but has to be treated with some caution under RF conditions.

\section{ACKNOWLEDGMENT}

The authors would like to thank OceanOptics and Mesuro Ltd., for technical assistance. They would also like to thank J.W. Pomeroy for performing thermal simulations on the devices for extracting the lattice temperature.

\section{References}

[1] J.-B. Fonder et al., "Compared deep class-AB and class-B ageing on $\mathrm{AlGaN} / \mathrm{GaN}$ HEMT in S-Band pulsed-RF operating life," Microelectron. Rel., vol. 52, no. 11, pp. 2561-2567, 2012, doi: 10.1016/j.microrel.2012.04.024.

[2] A. R. Barnes and F. Vitobello, "Preliminary reliability data from accelerated RF life tests on European GaN HEMTs," in Proc. CS-MANTECH, Boston, MA, USA, 2012, pp. 87-90.

[3] Y. Ohno, T. Nakao, S. Kishimoto, K. Maezawa, and T. Mizutani, "Effects of surface passivation on breakdown of $\mathrm{AlGaN} / \mathrm{GaN}$ high-electronmobility transistors," Appl. Phys. Lett., vol. 84, no. 12, pp. 2184-2186, 2004, doi: 10.1063/1.1687983.

[4] C. Hodges et al., "Optical investigation of degradation mechanisms in AlGaN/GaN high electron mobility transistors: Generation of nonradiative recombination centers," Appl. Phys. Lett., vol. 100, no. 11, p. 112106, Mar. 2012, doi: 10.1063/1.3693427.

[5] A. Sozza, C. Dua, E. Morvan, B. Grimber, and S. L. Delage, "A 3000 hours DC life test on AlGaN/GaN HEMT for RF and microwave applications," Microelectron. Rel., vol. 45, nos. 9-11, pp. 1617-1621, 2005, doi: 10.1016/j.microrel.2005.07.081.

[6] A. Chini et al., "Correlation between DC and RF degradation due to deep levels in AlGaN/GaN HEMTs," in IEDM Tech. Dig., Dec. 2009, pp. 1-4, doi: 10.1109/IEDM.2009.5424394.

[7] T. Brazzini et al., "Electroluminescence of hot electrons in $\mathrm{AlGaN} / \mathrm{GaN}$ high-electron-mobility transistors under radio frequency operation," Appl. Phys. Lett., vol. 106, p. 213502, May 2015, doi: $10.1063 / 1.4921848$

[8] T. Brazzini et al., "Study of hot electrons in AlGaN/GaN HEMTs under RF class $\mathrm{B}$ and class $\mathrm{J}$ operation using electroluminescence," Microelectron. Rel., vol. 55, pp. 2493-2498, Dec. 2015, doi: 10.1016/j.microrel.2015.09.023.

[9] S. Mukherjee, Y. Puzyrev, J. Chen, D. M. Fleetwood, R. D. Schrimpf, and S. T. Pantelides, "Hot-carrier degradation in GaN HEMTs due to substitutional iron and its complexes," IEEE Trans. Electron Devices, vol. 63, no. 4, pp. 1486-1494, Apr. 2016, doi: 10.1109/TED.2016.2532806.

[10] Y. S. Puzyrev, B. R. Tuttle, R. D. Schrimpf, D. M. Fleetwood, and S. T. Pantelides, "Theory of hot-carrier-induced phenomena in $\mathrm{GaN}$ high-electron-mobility transistors," Appl. Phys. Lett., vol. 96, no. 5, p. 053505 , 2010, doi: 10.1063/1.3293008.
[11] Y. S. Puzyrev et al., "Dehydrogenation of defects and hot-electron degradation in GaN high-electron-mobility transistors," J. Appl. Phys., vol. 109 , no. 3, p. 034501,2011 , doi: 10.1063/1.3524185.

[12] T. Brazzini et al., "Mechanism of hot electron electroluminescence in GaN-based transistors," J. Phys. D, Appl. Phys., vol. 49, no. 43, p. 435101, 2016, doi: 10.1088/0022-3727/49/43/435101.

[13] M. Meneghini, A. Stocco, R. Silvestri, G. Meneghesso, and E. Zanoni, "Degradation of $\mathrm{AlGaN} / \mathrm{GaN}$ high electron mobility transistors related to hot electrons," Appl. Phys. Lett., vol. 100, p. 233508, May 2012, doi: $10.1063 / 1.4723848$.

[14] F. Gütle et al., "Radiative inter-valley transitions as a dominant emission mechanism in AlGaN/GaN high electron mobility transistors," Semicond. Sci. Technol., vol. 27, no. 12, p. 125003, 2012, doi: 10.1088/ $0268-1242 / 27 / 12 / 125003$.

[15] M. Meneghini et al., "Extensive analysis of the luminescence properties of AlGaN/GaN high electron mobility transistors," Appl. Phys. Lett., vol. 97 , no. 6, p. 063508 , 2010, doi: 10.1063/1.3479917.

[16] M. Meneghini, G. Meneghesso, and E. Zanoni, "Analysis of the reliability of AlGaN/GaN HEMTs submitted to on-state stress based on electroluminescence investigation," IEEE Trans. Device Mater. Rel., vol. 13, no. 2, pp. 357-361, Jun. 2013, doi: 10.1109/TDMR.2013.2257783.

[17] M. A. Casbon, T. Brazzini, P. J. Tasker, M. Uren, and M. Kuball, "Simultaneous measurement of optical and RF behavior under $\mathrm{CW}$ and pulsed fully active harmonic load-pull," in Proc. 87th ARFTG Microw. Meas. Conf. (ARFTG), 2016, pp. 1-4, doi: 10.1109/ARFTG.2016.7501954.

[18] J. W. Pomeroy, M. J. Uren, B. Lambert, and M. Kuball, "Operating channel temperature in GaN HEMTs: DC versus RF accelerated life testing," Microelectron. Rel., vol. 55, no. 12, pp. 2505-2510, 2015, doi: 10.1016/j.microrel.2015.09.025.

[19] H. Sun, M. M. Bajo, M. J. Uren, and M. Kuball, "Implications of gateedge electric field in AlGaN/GaN high electron mobility transistors during OFF-state degradation," Microelectron. Rel., vol. 54, no. 12, pp. 2650-2655, 2014, doi: 10.1016/j.microrel.2014.09.020.

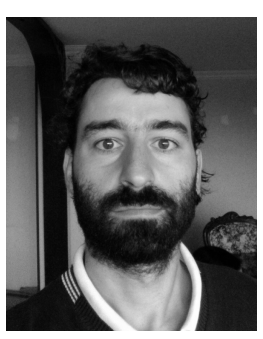

Tommaso Brazzini received the B.S. and M.S. degrees from the Universitá degli studi di Firenze, Florence, Italy, and the Ph.D. degree from the Instituto de Sistemas Optoelectrónicos y Microtecnología, Universidad Politecnica de Madrid, Madrid, Spain, with a focus on indium containing nitrides characterization and device application.

$\mathrm{He}$ is currently a Research Associate with the Centre for Device Thermography and Reliability, University of Bristol, Bristol, U.K.

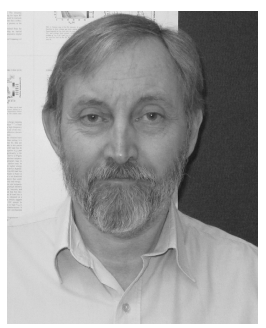

Michael A. Casbon was a Waveguide Engineer in Cambridge, U.K., initially designing TV broadcast channel diplexers and vision/sound combiners, and subsequently designed the first $1-\mathrm{KW}$ band $4 / 5$ broadband module for what became the Harris Sceptre solid state TV transmitter range. $\mathrm{He}$ became the Chief Engineer with Wessex Electronics, Holton Heath, U.K. He is currently a Researcher with Cardiff University, Cardiff, U.K., using active harmonic load-pull techniques to characterize semiconductor devices.

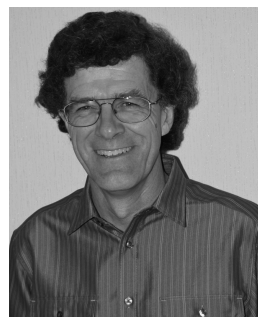

Michael J. Uren (M'06) received the M.A. and Ph.D. degrees in physics from the University of Cambridge, Cambridge, U.K. He was with RSRE, DERA, and QinetiQ, where he was involved in $\mathrm{Si}, \mathrm{SiC}$, and $\mathrm{GaN}$ devices. $\mathrm{He}$ is currently a Research Professor with the University of Bristol, Bristol, U.K. He is also a fellow of the Institute of Physics. 


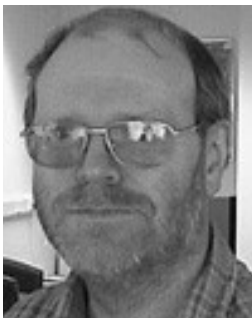

Paul J. Tasker (F'15) received the B.Sc. and Ph.D. degrees from Leeds University, Leeds, U.K., in 1979 and 1983, respectively.

He was with Lester Eastman, Cornell University, Ithaca, NY, USA, from 1984 to 1990 , as a Research Associate. He was a Senior Manager with the Fraunhofer Institute for Applied Solid State Physics from 1990 to 1995 . He joined Cardiff University, Cardiff, U.K., as a Professor in 1995.

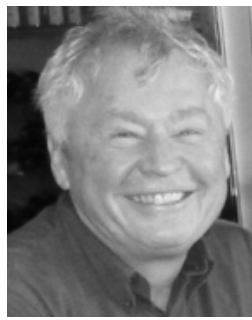

Helmut Jung received the Diploma degree from the University of Stuttgart, Stuttgart, Germany, in 1980, and the Ph.D. degree from the Max Planck Institute for Solid State Physics, Stuttgart, in 1984.

From 1984 he was scientist at the Daimler Chrysler Research Center in Ulm, Germany. Since 1996 he is at United Monolithic Semiconductors (UMS), presently responsible for technology co-operations.

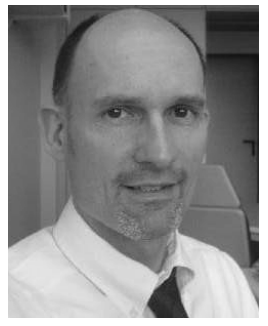

Hervé Blanck received the M.S. and Ph.D. degrees from the University of Strasbourg, Strasbourg, France, in 1986 and 1989, respectively.

$\mathrm{He}$ joined the Central Research Laboratory, Thomson-CSF, Orsay, France. In 1996, he joined United Monolithic Semiconductors, Ulm, Germany, with a focus on HBT development and industrialization, where he has been the Manager with the Technology Development Department since 2003.

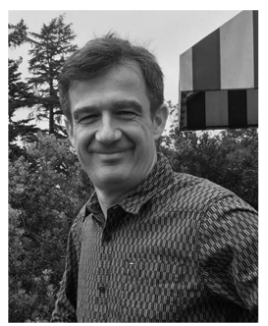

Martin Kuball (SM'09) received the Ph.D. degree from the Max Planck Institute for Solid State Research, Stuttgart, Germany.

$\mathrm{He}$ is currently a Professor in physics and the Director of the Center for Device Thermography and Reliability, University of Bristol, Bristol, U.K. He was the Feodor-Lynen Post-Doctoral Fellow with Brown University, Providence, RI, USA.

Dr. Kuball is a fellow of the IET and the IoP. 